\title{
A New Questionnaire for Quick Assessment of Food Tolerance after Bariatric Surgery
}

\author{
Michel Suter, MD, PD, FACS ${ }^{1,2}$; Jean-Marie Calmes, MD²; Alexandre \\ Paroz, MD²; Vittorio Giusti, MD, PD ${ }^{3}$
}

${ }^{1}$ Department of Surgery, Hôpital du Chablais, Aigle-Monthey, Switzerland; ${ }^{2}$ Department of Visceral Surgery, and ${ }^{3}$ Division of Endocrinology, Diabetology and Metabolism, Centre Hospitalier Universitaire Vaudois (CHUV), Lausanne, Switzerland

Background: Bariatric surgery is often associated with reduced food tolerance and sometimes frequent vomiting, which influence quality of life, but are not included in the overall evaluation of these procedures, notably the BAROS. Our aim was to develop a simple questionnaire to evaluate food tolerance during follow-up visits.

Methods: A one-page questionnaire including questions about overall satisfaction regarding quality of alimentation, timing of eating over the day, tolerance to several types of food, and frequency of vomiting/regurgitation was developed. A composite score was derived from this questionnaire, giving a score of 1 to 27 . Validation was performed with a group of nonobese adults and a group of morbidly obese nonoperated patients. Patients were administered the questionnaire at follow-up visits since January 1999. Data were collected prospectively.

Results: It takes 1-2 minutes to fill out the questionnaire. Food tolerance is worse in the morbidly obese population compared with non-obese adults (24.2 vs 25.2, $P=0.004)$. Following Roux-en-Y gastric bypass, food tolerance is reduced after 3 months (21.2), but becomes comparable to that of the normal population and remains so at 1 year postoperatively. Following gastric banding, food tolerance is already significantly reduced after 3 months (22.3), and worsens continuously over time (19.03 after 7 years). In the gastric banding population, the decision to adjust the band is based at least partially on food tolerance, and the questionnaire proved helpful in that respect.

Conclusions: Our new questionnaire proved very easy to use, and helpful in day-to-day practice, especially after gastric banding. It was also helpful in comparing food tolerance over time after surgery, and in

Correspondence to: PD Dr. M. Suter, Department of Surgery, Hôpital du Chablais, 1860 Aigle, Switzerland. Fax +41-24-46886-23; e-mail: michelsuter@ netplus.ch comparing food tolerance between procedures. Evaluation of food tolerance should be part of the overall evaluation of the results after bariatric surgery.

Key words: Morbid obesity, bariatric surgery, gastric bypass, gastric banding, food tolerance, quality of life

\section{Introduction}

Because of the increasing prevalence of morbid obesity in all western countries, the number of bariatric operations performed each year is growing rapidly. Traditionally, the results of the different procedures have mostly been evaluated on the basis of weight loss. In 1998, the Bariatric Analysis and Reporting Outcome System (BAROS) score was developed and proposed as a standard tool to assess results of bariatric surgery. ${ }^{1}$ This composite score considers excess weight loss, quality of life improvement, correction of co-morbidities and complications. Even in its latest form, ${ }^{2}$ it does not allow, however, the evaluation of one major difference between purely restrictive procedures such as gastric banding (GB) or vertical banded gastroplasty (VBG), and others (Roux-en-Y gastric bypass (RYGBP) and biliopancreatic diversion), namely food tolerance. This limitation has been recognized by others, ${ }^{3}$ but so far has not been addressed in a satisfying manner.

When planning our prospective randomized study ${ }^{4}$ comparing the results of GB with the Lapband ${ }^{\circledR}$ or the $\mathrm{SAGB}^{\circledR}$, and because we believed that the physical properties of the various types of gas- 
tric bands might account for differences in food tolerance, we devised a questionnaire in 1998 to assess food tolerance in a simple but standardized manner during follow-up visits. In this paper, we present this questionnaire for the first time, with examples of applications in the clinical setting.

\section{Materials and Methods}

This one-page questionnaire (see Appendix 1 at end of article) is divided in four parts: 1) an overall assessment of the patient's satisfaction about the quality of his/her alimentation, 2) questions about the timing of meals and food intake between meals, 3 ) an evaluation of tolerance of eight different types of food, and 4) an evaluation of the frequency of vomiting / regurgitation. On the basis of the answers, the physician can ask further questions about specific topics, like amount of food eaten during meals, quality and frequency of snacks or "grazing". A score is derived from parts 1, 3 and 4 of the questionnaire. Patient's satisfaction about food intake is given between 1 (very poor) and 5 (excellent) points. Food tolerance is given between 0 and 16 points: for each specific type of food, 2 points if the patient can eat this type without any particular difficulty, 1 point if he/she can eat it with some difficulties/restrictions, and 0 points if he/she cannot eat it at all. The importance of vomiting/regurgitation is given between 0 and 6 points: daily vomiting or regurgitation -0 points, three or more times a week -2 points, up to twice a week -4 points, never -6 points. The score can therefore vary between 1 and 27, 27 being the maximum for an excellent food tolerance.

All data was collected prospectively. The questionnaire was administered to the patients quarterly during the 1st postoperative year, biannually from the 2 nd to the 5 th postoperative year, and annually thereafter. Over 300 patients were evaluated in this way after GB, and more than 600 after RYGBP. The questionnaire was also administered to a group of 75 non-obese volunteers for validation in the normal population, and to a group of 55 non-operated morbidly obese patients. After gastric banding, only patients who still had the band in place were considered for evaluation. Patients in whom the band had been removed, or who had been converted to anoth- er bariatric procedure were excluded from analysis.

Statistical analysis was performed using the student's $t$-test for continuous variables, and with the $\chi^{2}$-test for categorical variables. A value of $P<0.05$ was considered significant.

\section{Results}

The questionnaire proved easy to fill in for the patients, who required usually $<2$ minutes. In the normal population, the mean score was 25.2 , with $86.6 \%$ of the respondents having a score $\geq 24$ (Figure 1). In the non-operated morbidly obese patients (Figure 2), the mean score was 24.2, which was significantly lower than in the normal population ( $P=0,004$, Student's $t$-test). Only $63.6 \%$ of the morbidly obese patients had a score $\geq 24\left(\chi^{2}=9.45\right.$, $P=0.002)$. These differences were mostly due to a higher prevalence of patients who were dissatisfied about the quality of their alimentation (part 1 of the questionnaire) in the morbidly obese group $\left(\chi^{2}=7.18, P=0.007\right)$.

After GB, food tolerance was significantly altered. The mean score was significantly much lower than in the normal population at all time intervals, with a slow, but continuous, decrease over time (Figure 3 ). After 3 and 5 years, only 16.5 , respectively $17.2 \%$ of the patients had a score $\geq 24$ ( $P<0,001$ versus normal population). There was no significant difference

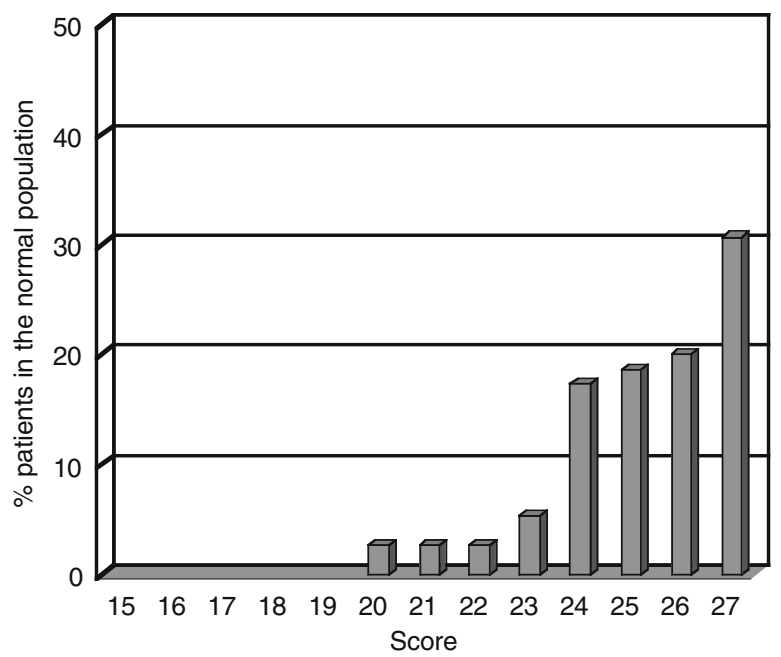

Figure 1. Distribution of the food tolerance score in a group of 75 normal non-obese volunteers. 


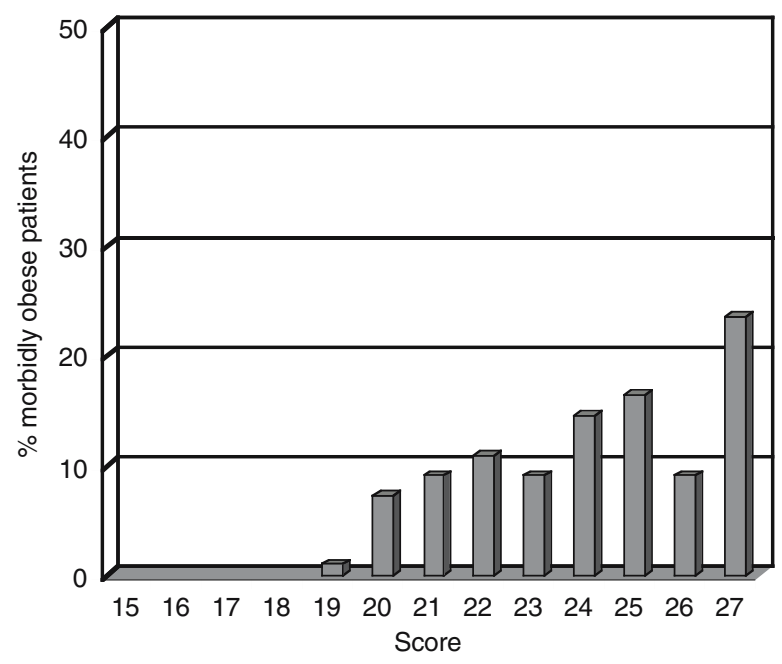

Figure 2. Distribution of the food tolerance score in a group of 55 non-operated morbidly obese patients.

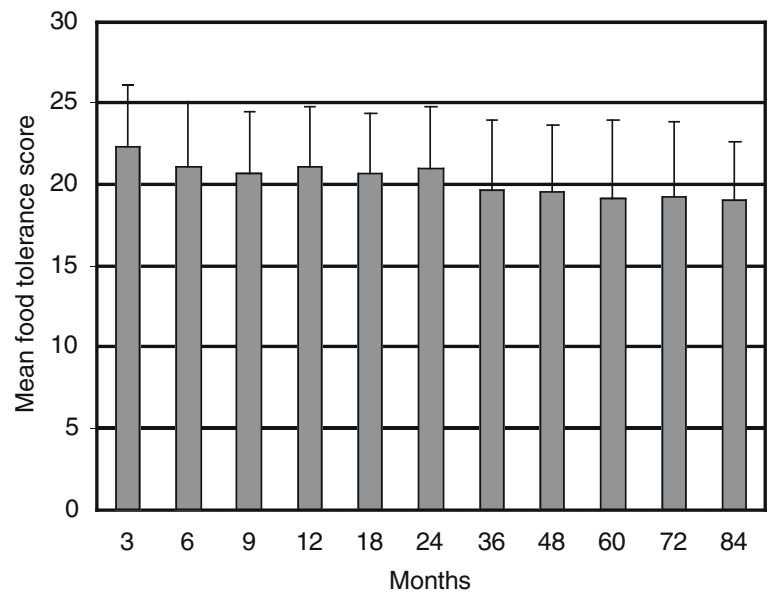

Figure 3. Evolution of the food tolerance score after gastric banding.

between patients with a Lap-band ${ }^{\circledR}$ (Allergan, Santa Barbara, CA, USA) and patients with a $\mathrm{SAGB}^{\circledR}$ (Obtech, Ethicon Endo-Surgery) (Figure 4).

After RYGBP, food tolerance was impaired during the 1st postoperative year, especially during the first semester, but returned to normal later on, and remained comparable to that of the normal population until the 5th postoperative year (Figure 5). The proportion of patients with a score $\geq 24$ was $31.4 \%$ after 3 months, $58.9 \%$ after 6 months, and increased to $80.1 \%$ after the 1 st postoperative year. It remained stable later on, at $86.8 \%$ after 3 years and $78 \%$ after 5 years.

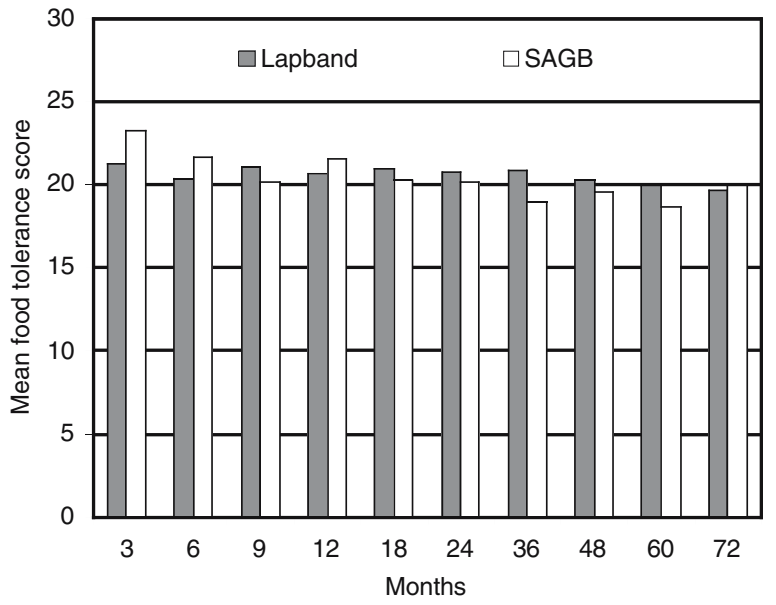

Figure 4. Comparison of food tolerance after gastric banding between the Lap-band ${ }^{\circledR}$ and the SAGB ${ }^{\circledR}$.

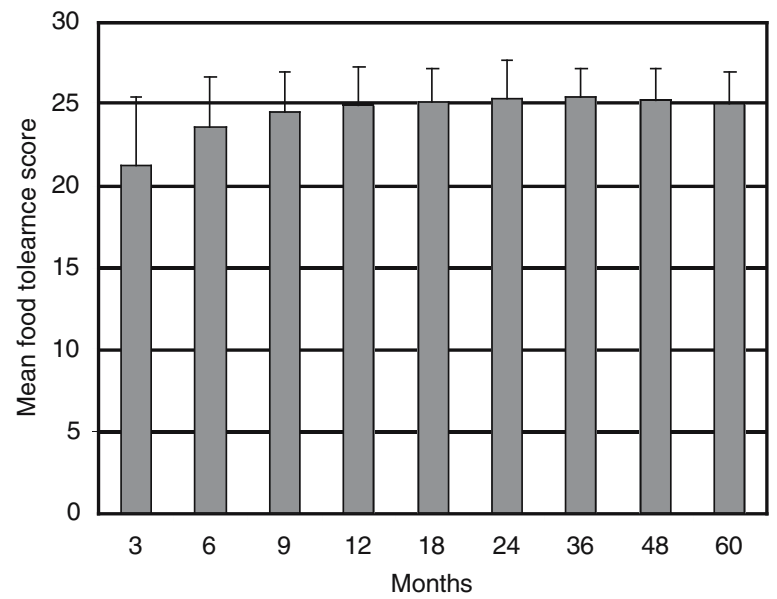

Figure 5. Evolution of the food tolerance score after RYGBP.

Comparison between patients after GB and RYGBP showed that, except during the first postoperative trimester, food tolerance was significantly much better after RYGBP, and remained so in the long term (Figure 6). The difference between the two procedures even increased with time, despite the fact that patients with a particularly poor food tolerance and/or complications after GB were progressively converted to RYGBP and therefore excluded from analysis. The distribution of the scores was also strikingly different: very few patients had a low score after RYGBP, and none a score $<20$, compared with $44.2 \%$ of the patients who had a score $<20$ after 


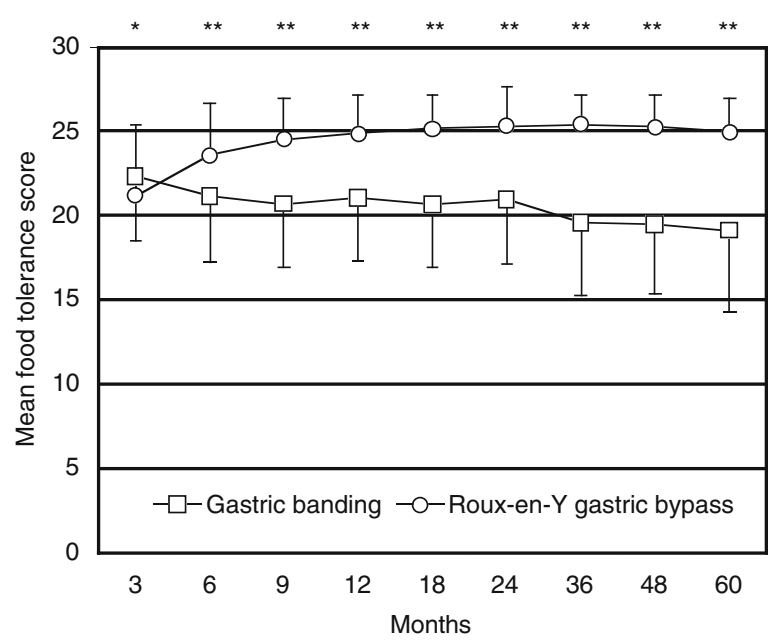

Figure 6. Comparison of food tolerance between gastric banding and gastric bypass over the first 5 postoperative years. ${ }^{*} P<0.05,{ }^{* *} P<0.001$.

GB at 3 years postoperatively $(P<0,001)$, and 43.9 at 5 years $(P<0.001)$ (Figure 7$)$.

\section{Discussion}

Quality of life includes several parameters regarding physical, mental, and social well being, among others. The capacity to eat and enjoy a variety of aliments not only alone, but also in society, certainly accounts among factors that can alter quality of life. Several bariatric procedures induce a pure alimentary restriction that does not only limit the quantity of food intake, but qualitatively interferes with the variety of aliments that can be ingested. Due to these limitations, patients often slowly switch to a soft semi-liquid diet after restrictive surgery, in order to be able to eat "normal meals" in public without having to leave the table repeatedly to regurgitate or vomit. So far, food restriction has not been evaluated in a standardized manner after bariatric surgery. It is not included in the recognized scoring systems, including the BAROS, although it can significantly alter the overall quality of life of patients.

Dysphagia questionnaires have been elaborated to assess disorders that cause dysphagia, such as achalasia. ${ }^{5-7}$ To our knowledge, no study has ever reported results in bariatric patients using this type of instrument.

On the other hand, several authors have used the

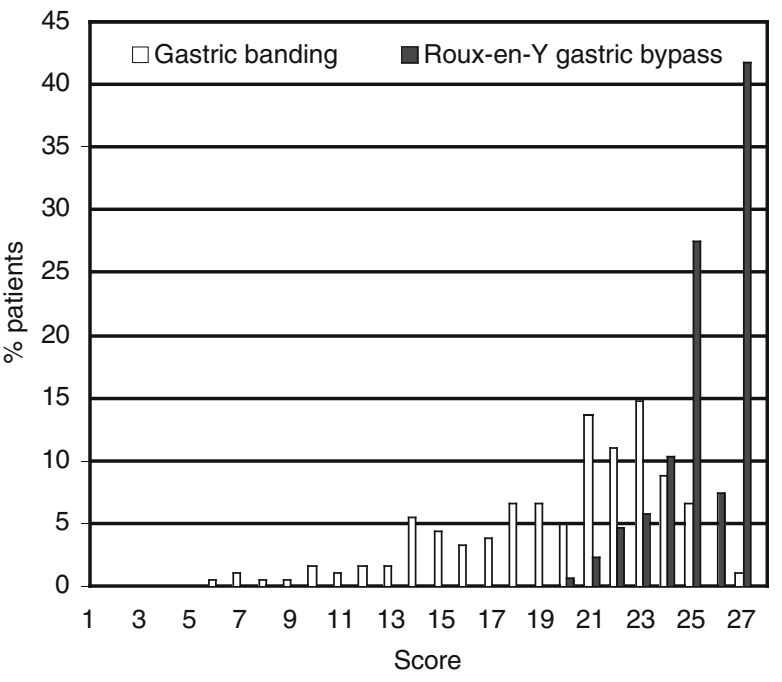

Figure 7. Distribution of food tolerance scores in gastric banding and RYGBP patients after 36 months.

Gastrointestinal Quality of Life Index (GIQLI) ${ }^{8}$ to assess quality of life in morbidly obese non-operated patients, as well as after various bariatric procedures. This score has been developed to include digestive symptoms in the overall assessment of quality of life in various digestive diseases. Using this score, Poves et $\mathrm{al}^{9}$ not surprisingly found a reduced quality of life in morbidly obese patients. The same authors recently showed that the quality of life of morbidly obese patients, as measured by the GIQLI, improved significantly after laparoscopic RYGBP, and was almost similar to that of non-obese control subjects, including the subscore regarding the disease-specific symptoms. ${ }^{10}$ Lee at al ${ }^{11}$ used the GIQLI to assess quality of life after various bariatric procedures. After VBG, they found that the GIQLI increased 6 months after surgery, but returned to baseline after 2 years, due to worsening of the digestive symptoms. ${ }^{11}$ Comparing VBG with RYGBP, they showed that patients with VBG enjoyed eating less than their RYGBP counterparts, with a greater sensation of restriction and more dysphagia. ${ }^{12}$ Comparing RYGBP with the mini-gastric bypass, they showed an overall improvement in quality of life after both procedures without worsening of digestive specific symptoms in either of them. ${ }^{13}$ More recently, they showed that, 2 years after GB, patients enjoyed eating less than before surgery, and had more nausea, dysphagia and regurgitations, with an overall significant worsening of the symp- 
toms score. ${ }^{14}$ Freys et a ${ }^{15}$ also evaluated patients after GB with the GIQLI; up to 18 months postoperatively, they found a significant overall improvement in quality of life, but no difference in the symptoms score. We have no explanation for these differences between the two studies. Clinical experience clearly suggests that the different bariatric procedures do not affect digestive symptoms and food intake in the same manner. The above results from the literature seem to substantiate this impression. Restrictive surgery tends to worsen the GIQLI subscore on digestive symptoms, which RYGBP does not.

The new questionnaire presented in this paper is simple to fill out, reproducible, and allows for comprehensive comparisons over time in the individual as well as among different groups of patients. Most normal volunteers have a very good score, and those with a lower score usually are dissatisfied with the overall quality of their alimentation because they do not have enough time to eat at lunchtime, or because the variety of meals offered is not wide enough. Because they are less satisfied with their overall alimentation, morbidly obese patients have a lower mean score than non-obese normal volunteers. This is not surprising, and reflects the frustration of these patients, who have usually tried several diets that they cannot comply with in the long term, and at least partly feel responsible for the worsening of their condition.

Our results show that food tolerance is considerably altered even early after GB, with a continuous and slow worsening over time. The true effect is most probably worse than our figures show, because patients with the worst side-effects and tolerance after GB are progressively converted to RYGBP and therefore excluded from analysis. Contrary to what we believed when we initiated our randomized study comparing the high-pressure Lap-band ${ }^{\circledR}$ system with the low-pressure $\mathrm{SAGB}^{\circledR}$, we found no significant difference in long-term food tolerance between these two devices. ${ }^{4}$ This reflects the fact that the diameter of the stoma is more important for the level of food restriction than the pressure generated within the device by band adjustment. It is likely that other bands will generate the same restriction and therefore the same worsening of food tolerance over time as the bands tested in our experience. Clearly, the tightness of the band has a marked effect on food tolerance. Very poor food tolerance after GB should prompt adjustment towards a larger stoma. The price for better food tolerance, however, is usually weight regain, which is often not accepted by the patients, and the aim here is to find an acceptable compromise, which is not always possible. On the contrary, RYGBP does not alter food tolerance, except during the early postoperative period, when restriction is maximal due to scarring and postoperative edema. Our results clearly show that RYGBP offers better long-term food tolerance than GB as of the end of the 1st postoperative year. Our patients who underwent GB first, and later RYGBP also substantiate this difference, and all claim that food tolerance is better after RYGBP. Most would have chosen RYGBP first in retrospect, better food tolerance being one of their major reasons.

Our questionnaire can also be used in the individual patient to assess food tolerance and eating behavior at regular follow-up visits. Ideally, weight loss should be optimal with a good food tolerance. After GB, if food tolerance is good but weight loss insufficient, the band can easily be tightened. In patients with poor food tolerance, the band is usually too tight, or there is a complication, such as band slippage or esophageal dilatation, and the band must be loosened. Poor food tolerance is the reason why so many patients eventually switch to a high-calorie semi-liquid diet, with its resultant paradoxical weight gain. A sudden improvement in food tolerance, especially if accompanied by weight regain, suggests a leak in the system, or band erosion. In patients with RYGBP, a sudden decrease in food tolerance usually suggests a complication such as marginal ulcer.

In conclusion, the new questionnaire presented in this paper appears reliable, easy to use, patientfriendly, and useful both in following-up individuals after bariatric surgery, and in comparing results after different procedures as part of the evaluation of quality of life. If teams with an interest in these specific aspects of bariatric surgery validate it with results similar to ours, it could become a routine tool in the evaluation of bariatric patients, with points deducted from the BAROS score in patients with poor food tolerance. This would allow a single instrument to be used for all types of bariatric procedures, and obviate the need for a modified score for restrictive surgery. ${ }^{3}$ 


\section{References}

1. Oria HE, Moorehead MK. Bariatric analysis and reporting outcome system (BAROS). Obes Surg 1998; 8: 487-99.

2. Moorehead MK, Ardelt-Gattinger E, Lechner $\mathrm{H}$ et al. The validation of the Moorehead-Ardelt quality of life questionnaire II. Obes Surg 2003; 13: 684-92.

3. Nini E, Slim K. The need for a modified BAROS for gastric banding. Obes Surg 2004; 14: 147.

4. Suter M, Giusti V, Worreth M et al. Laparoscopic gastric banding. A prospective randomised study comparing the Lap-band ${ }^{\circledR}$ and the SAGB ${ }^{\circledR}$ : Early results. Ann Surg 2005; 241: 555-62.

5. Perrone JM, Frisella MM, Desai KM et al. Results of laparoscopic Heller-Toupet operation for achalasia. Surg Endosc 2004; 18: 1565-71.

6. Rossetti G, Brusciano L, Amato G et al. A total fundoplication is not an obstacle to esophageal emptying after Heller myotomy for achalasia. Ann Surg 2005; 241: 614-21.

7. Johnson LF, DeMeester TR. Twenty-four hour pHmonitoring of the distal esophagus: a quantitative measure of gastroesophageal reflux. Am J Gastroenterol 1974; 62: 325-32.

8. Eypasch E, Williams JI, Wood-Dauphinee S et al.
Gastrointestinal Quality of Life Index. Development, validation and application of a new instrument. $\mathrm{Br} \mathrm{J}$ Surg 1995; $82: 216-22$.

9. Poves I, Macías GJ, Cabrera M et al. Quality of life in morbid obesity. Rev Esp Enferm Dig 2005; 97: 187-95.

10. Poves I, Cabrera M, Maristany C et al. Gastrointestinal quality of life after laparoscopic Roux-en-Y gastric bypass. Obes Surg 2006; 16: 19-23.

11.Lee WJ, Yu PJ, Wang W et al. Gastrointestinal quality of life following laparoscopic vertical banded gastroplasty. Obes Surg 2002; 12: 819-24.

12.Lee WJ, Huang MT, Yu PJ et al. Laparoscopic vertical banded gastroplasty and laparoscopic gastric bypass: a comparison. Obes Surg 2004; 14: 626-34.

13. Lee WJ, Yu PJ, Wang W et al. Laparoscopic Roux-en$\mathrm{Y}$ gastric bypass versus mini-gastric bypass for the treatment of morbid obesity. A prospective randomized controlled clinical trial. Ann Surg 2005; 242: 20-8.

14. Lee WJ, Wang W, Yu PJ et al. Gastrointestinal quality of life following laparoscopic adjustable gastric banding in Asia. Obes Surg 2006; 16: 586-91.

15. Freys SM, Tigges H, Heimbucher J et al. Quality of life following laparoscopic gastric banding in patients with morbid obesity. J Gastrointest Surg 2001; 5: 401-7.

(Received September 23, 2006; accepted December 2, 2006) 
Suter et al

\section{Appendix 1.}

\section{QUALITY OF ALIMENTATION}

Name: Surname: Months after Surgery: months

How would you rate your overall satisfaction regarding how you can eat presently?

Excellent
Good
Acceptable
Poor
Very Poor

$\square$
$\square$
$\square$
$\square$
$\square$

Why?

How many meals do you eat a day?

Among the following meals, which one do you have?

Breakfast

Lunch

Supper

Which of them constitutes your daily main meal?

Do you eat between meals?

Yes

No

$\square$

If yes, when?

Morning

Afternoon

Evening

Can you eat everything?

Yes

No

More specifically, how can you eat?

$\begin{array}{lllll}\text { Red meat } & \square \text { Easily } & \square & \text { With some difficulties } & \square \text { Not all } \\ \text { White meat } & \square & \square & \square \\ \text { Salad } & \square & \square & \square \\ \text { Vegetables } & \square & \square & \square \\ \text { Bread } & \square & \square & \square \\ \text { Rice } & \square & \square & \square \\ \text { Pasta } & \square & \square & \square \\ \text { Fish } & \square & \square & \square\end{array}$

Are there other types of food that you cannot eat at all?

Do you vomit/regurgitate? $\quad \square$ Daily $\quad \square$ Often (> 2x/week) $\quad \square$ Rarely $\quad \square$ Never 